\title{
Synthesis and antifungal activity of novel pyrazolines and isoxazolines derived from cuminaldehyde
}

\author{
Tao Zhang, ${ }^{1, *}$ Mengya Dong, ${ }^{2}$ Jianjiang ZhaO, \\ Xiaofang $\mathrm{ZHANG}^{1}$ and Xiangdong $\mathrm{MeI}^{2}$,* \\ ${ }^{1}$ Institute of Plant Protection, Hebei Academy of Agriculture and Forestry \\ Sciences, Integrated Pest Management Center of Hebei Province, \\ Key Laboratory of IPM on Crops in Northern Region of North China, \\ Ministry of Agriculture, Baoding 071000, P.R. China \\ ${ }^{2}$ State Key Laboratory for Biology of Plant Diseases and Insect Pests, \\ Institute of Plant Protection, Chinese Academy of Agricultural Sciences, \\ Beijing 100193, P.R. China
}

(Received April 11, 2019; Accepted May 15, 2019)

Two series of novel cuminaldehyde derivatives containing pyrazoline and isoxazoline moieties have been designed and synthesized. All of the compounds were characterized via ${ }^{1} \mathrm{H}-\mathrm{NMR},{ }^{13} \mathrm{C}-\mathrm{NMR}$, and HRMS. The antifungal activities were evaluated against six plant-pathogenic fungi. 3-(2-Fluorophenyl)-5-(4-isopropylphenyl) isoxazoline (2d) and 1-acetyl3-(2-fluorophenyl)-5-(4-isopropylphenyl)-2-pyrazoline (3d) displayed higher antifungal activities than commercial fungicides against Sclerotinia sclerotiorum, Physalospora piricola and Pyricularia oryzae. The title compounds (2d and 3d) with strong antifungal activities are worth being further evaluated in vivo and in the field. (c) Pesticide Science Society of Japan

Keywords: cumin, cuminaldehyde, pyrazoline, isoxazoline, antifungal activity.

Electronic supplementary materials: The online version of this article contains supplementary materials (Supplemental Tables S1 and S2; NMR spectral data), which is available at http://www.jstage.jst.go.jp/browse/ jpestics/

\section{Introduction}

Cuminaldehyde, the main composition of an essential oil extracted from Cuminum cyminum seeds, has been the subject of extensive concern because of its wide range of bioactivities. ${ }^{1-4)}$ Notably, it has been reported to be the key antioxidant and antimicrobial component in plant essential oils. ${ }^{5-7)}$ In recent years, novel cuminaldehyde derivatives have been designed and synthesized, and many have showed antifungal and antioxidant activities. For example, Peixoto et al. reported a series of novel

\footnotetext{
* To whom correspondence should be addressed.

E-mail: cauzht@163.com,xdmei@ippcaas.cn

Published online July 25, 2019

(C) Pesticide Science Society of Japan
}

compounds derived from cuminaldehyde that showed antifungal activities against Candida strains. $\left.{ }^{8}\right)$ Sid et al. reported 1-formyl-3-phenyl-5-(4-isopropylphenyl)-2-pyrazoline as an antifungal chemical against Aspergillus niger. ${ }^{9)}$

Heterocyclic nitrogenous compounds have received considerable attention due to their significant bioactivities. ${ }^{10)}$ Isoxazoline and pyrazoline, well known as important five-membered heterocyclic nitrogenous scaffolds, have been widely applied as pharmaceuticals and agrochemicals because of their antifungal activities. Fluralaner, a commercial insecticide, is a successful example that introduces isoxazoline as the scaffold. ${ }^{11)}$ Many studies have also focused on the antifungal activities of isoxazoline derivatives. ${ }^{12)}$ As for pyrazoline, many kinds of pyrazoline derivatives, especially $N$-acetylated pyrazoline derivatives, have been reported to show significant antifungal activities.,13-16)

Concerning the synthesis of isoxazolines and pyrazolines, the methods with stable and easily accessed starting materials and those conducted under mild conditions are more acceptable. ${ }^{15)}$ Usually, the sophisticated way to synthesize isoxazoline and pyrazoline is by the cyclization of $\alpha, \beta$-unsaturated carbonyl compounds (chalcones) with hydroxylamine and hydrazine. ${ }^{13)}$ Chalcones can be easily obtained from a simple Claisen-Schmidt reaction. ${ }^{13)}$ Other technology has also been used to synthesize isoxazoline, such as visible-light catalysis and microwave-assisted synthesis. ${ }^{11,17)}$

Keeping the above observations in view, we designed 24 compounds by introducing a cuminaldehyde structure into an isoxazoline or pyrazoline scaffold, which will be expected to exhibit higher fungicidal activities through the coexistence of two kinds of pharmacophores.

\section{Materials and Methods}

\section{General}

All used reagents and solvents were purchased from SigmaAldrich and used without further purification. The synthesized compound structures were confirmed by ${ }^{1} \mathrm{H}-\mathrm{NMR},{ }^{13} \mathrm{C}-\mathrm{NMR}$ and HRMS. ${ }^{1} \mathrm{H}-\mathrm{NMR}$ and ${ }^{13} \mathrm{C}-\mathrm{NMR}$ spectra were recorded on a Bruker DPX300 NMR instrument $(300 \mathrm{MHz})$, using tetramethylsilane as an internal standard and $\mathrm{CDCl}_{3}$ or DMSO- $d_{6}$ as a solvent. HRMS spectra were obtained with an Agilent 6500 Series LC/Q-TOF-ESI-MS/MS. Melting points were performed with an RY-1G melting-point apparatus and were uncorrected. Analytical thin-layer chromatography (TLC) was performed on precoated plates, and spots were visualized under ultraviolet light (254 nm).

\section{Synthesis}

2.1. Preparation of $\beta$-unsaturated chalcones (1a-1l)

In this study, $\alpha, \beta$-unsaturated chalcones 1 were synthesized by using a Claisen-Schmidt reaction. ${ }^{9,14-16)}$ To a solution of substi- 


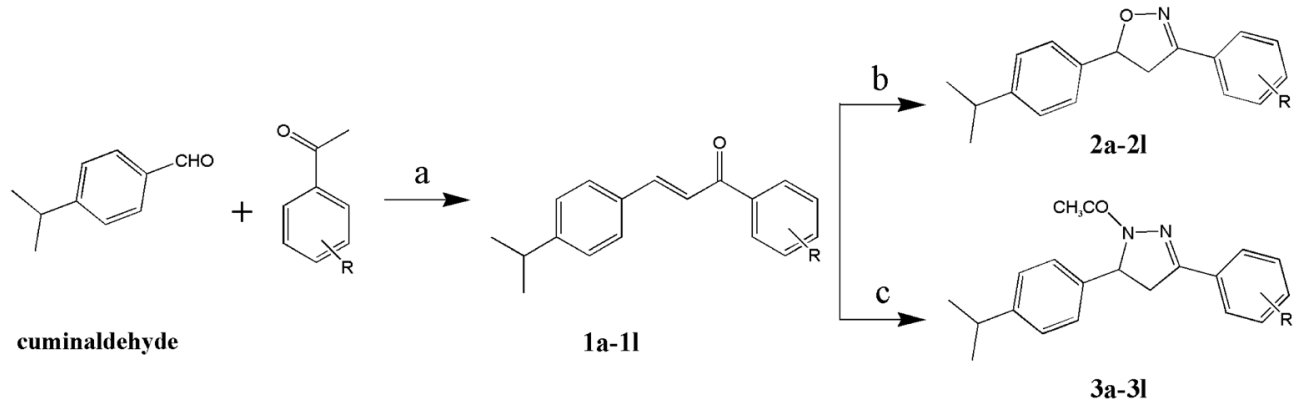

Scheme 1. Synthesis of cuminaldehyde derivatives. Reagents and conditions: (a) EtOH, $1 \mathrm{M} \mathrm{NaOH}, \mathrm{rt}, 8 \mathrm{hr}, 60-85 \%$; (b) EtOH, $\mathrm{NH} \mathrm{H}_{2} \mathrm{OH} \cdot \mathrm{HCl}, \mathrm{NaOH}, \mathrm{re}-$ flux, $8 \mathrm{hr}, 49-67 \%$; (c) acetic acid, $\mathrm{N}_{2} \mathrm{H}_{4} \cdot \mathrm{H}_{2} \mathrm{O}$, reflux, $4 \mathrm{hr}, 60-80 \%$. $\mathrm{R}=o-\mathrm{CH} 3(\mathbf{1 a}, \mathbf{2 a}, 3 \mathbf{3 a}) ; o-\mathrm{Br}(\mathbf{1 b}, \mathbf{2 b}, 3 \mathbf{3 b}) ; o-\mathrm{Cl}(\mathbf{1 c}, 2 \mathrm{2c}, 3 \mathbf{3 c}) ; o-\mathrm{F}(\mathbf{1 d}, \mathbf{2 d}, \mathbf{3 d}) ; m-\mathrm{CH}(\mathbf{1 e}, \mathbf{2 e}$,

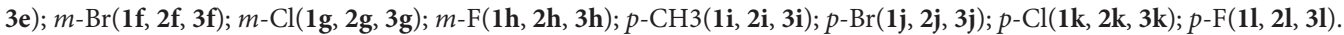

tuted acetophenone $(20 \mathrm{mmol})$ and cuminaldehyde $(20 \mathrm{mmol})$ in $15 \mathrm{~mL}$ of ethanol, $0.5 \mathrm{~mL}$ of $1 \mathrm{~mol} \cdot \mathrm{L}^{-1} \mathrm{NaOH}$ solution was added drop by drop. The mixture was stirred overnight at room temperature, and then the solvent was removed under reduced pressure. Chalcones $\mathbf{1}$ were obtained by recrystallization in ethanol.

\subsection{Preparation of 3-(substituted phenyl)-5-(4-isopropylphe-} $n y l)$ isoxazoline $(2 a-2 l)$

To a solution of chalcones $\mathbf{1}$ ( $5 \mathrm{mmol}$ ) and hydroxylamine hydrochloride $(5 \mathrm{mmol})$ in $30 \mathrm{~mL}$ of ethanol, $0.5 \mathrm{~mL}$ of $\mathrm{NaOH}$ $\left(1 \mathrm{~mol} \cdot \mathrm{L}^{-1}\right)$ was added drop by drop. The mixture was stirred at room temperature for $1 \mathrm{hr}$ and later refluxed for $8 \mathrm{hr}$. Subsequently, the reaction mixture was cooled and poured onto crushed ice, and then formed solids were collected by filtration and washed with cold water to obtain crude products of $\mathbf{2 a - 2 1}$. After purification by silica gel column chromatography, products were characterized via ${ }^{1} \mathrm{H}-\mathrm{NMR},{ }^{13} \mathrm{C}-\mathrm{NMR}$, and HRMS.

2.3. Preparation of 1-acetyl-3-(substituted phenyl)-5-(4isopropylphenyl)-2-pyrazoline ( $3 a-3 l)$

A mixture of chalcones $\mathbf{1}(5 \mathrm{mmol})$ and hydrazine hydrate $(5 \mathrm{mmol})$ in acetic acid $(15 \mathrm{~mL})$ was refluxed for $4 \mathrm{hr}$. After completion of the reaction (checked by TLC), the reaction mixture was cooled. Then the solid precipitation was filtered out, washed with cold water and acetone, and dried to gain crude products of 3a-31. Further purification was conducted by recrystallization in ethanol, and the products were characterized via ${ }^{1} \mathrm{H}-\mathrm{NMR}$, ${ }^{13} \mathrm{C}-\mathrm{NMR}$, and HRMS.

\section{Antifungal activity assay}

Tested strains (Sclerotinia sclerotiorum, Rhizoctonia solani, Botrytis cinerea, Physalospora piricola, Pyricularia oryzae and Colletotrichum lagenarium) were obtained from the Institute of Plant Protection, Chinese Academy of Agricultural Sciences, and were kept in the Agricultural Culture Collection of China (Preservation numbers: ACCC 30096, ACCC 30089, ACCC 30387, BNCC112323, ACCC 37660, and ACCC 30016, respectively). The fungal strains were maintained in a potato dextrose agar medium (PDA) medium and stored at $4^{\circ} \mathrm{C}$.

Evaluation of the fungicidal activities was performed according to reported protocols. ${ }^{18)}$ Preliminary determination of the inhibitory activities against six plant-pathogenic fungi was tested in vitro using the mycelia growth test on a PDA medium. Briefly, title compound solutions (in DMSO) and sterile molten PDA were mixed to obtain a final concentration of $200 \mu \mathrm{g} \cdot \mathrm{mL}^{-1}$ (containing 5\% DMSO). Then the mixture was poured into $90 \mathrm{~mm}$ Petri dishes $\left(15 \mathrm{~mL} \cdot \mathrm{dish}^{-1}\right)$, on which $5 \mathrm{~mm}$ mycelial disks of the six fungi were planted in the center. Three replicates were conducted for each treatment. DMSO (5\% contained in PDA) was used as a negative control, while thiabendazole and azoxystrobin were tested as positive controls. After a certain incubation period at $25^{\circ} \mathrm{C}$ (until the colonies in the control treatments had covered two-thirds of the Petri dishes), the mycelial growth diameters were measured using the cross-bracketing method, and the percentage of mycelial growth inhibition was then calculated. The inhibition percentages were calculated with the following equation: $I=[(C-T) /(C-5)] \times 100 \%$, where $T$ is the mycelial diameter $(\mathrm{mm})$ in the Petri dishes with compounds and $C$ is the diameter $(\mathrm{mm})$ of the DMSO control.

Furthermore, the antifungal activities of higher effective compounds (according to the preliminary assay) were evaluated using the inhibition percentages of tested compounds in the final series concentration. The $\mathrm{EC}_{50}$ values were calculated by linear-regression analysis.

\section{Results and Discussion}

\section{Chemistry}

The reaction sequences for the synthesis of 3 -(substituted phenyl)-5-(4-isopropylphenyl) isoxazoline (2a-2l) and 1-acetyl3-(substituted phenyl)-5-(4-isopropylphenyl)-2-pyrazoline (3a31) are outlined in Scheme 1 according to the reported method. ${ }^{19,20)}$

The intermediate chalcones, (E)-1-(4-isopropylphenyl)3-(substituted phenyl)prop-2-en-1-ones (1), a kind of $\alpha, \beta$-unsaturated ketones, were prepared by reacting the cuminaldehyde with substituted acetophenones via a facile procedure in the presence of a base with $60-85 \%$ yield. The chalcones were then converted into the isoxazoline and pyrazoline derivatives through different reactions. Isoxazoline derivatives $\mathbf{2 a}-\mathbf{2 l}$ were prepared by refluxing a mixture of chalcones, hydroxylamine hydrochloride, and $\mathrm{NaOH}$ in an ethanol medium 
with $49-67 \%$ yields. Compounds 1 and 2 were separated using column chromatography (ethyl acetate-petroleum ether). Monitoring via TCL, the positions of compounds $\mathbf{2 a - 2 d}$ and their byproducts were too close to be easily isolated. Thus, a less polar mobile phase was used to isolate compounds with lower yields. Pyrazoline derivatives $\mathbf{3 a}$-31 were synthesized by refluxing compounds 1 and hydrazine hydrate in acetic acid, then purified by recrystallization (in ethanol) with $60-80 \%$ yields. To analyze the relationship between structure and activity, unsubstituted isoxazoline (2-U) and pyrazoline (3-U) were also synthesized via a similar method.

All title compounds were confirmed by NMR and HRMS, and structural analysis data were shown as supplementary data. Additionally, the crystal structure of compound $\mathbf{3} \mathbf{g}$ was determined to further verify the structure of the synthetic compound in our previous study. ${ }^{21)}$

\section{Antifungal Activity in vitro}

As shown in Supplemental Table S1, preliminary determination of the inhibition activities of title compounds $\left(200 \mu \mathrm{g} \cdot \mathrm{mL}^{-1}\right)$ against six plant-pathogenic fungi (S. sclerotiorum, R. solani, $B$. cinerea, Ph. piricola, Py. oryzae, and C. lagenarium) suggested that all of the compounds showed significant antifungal activities against plant-pathogenic fungi, with an inhibition rate higher than $50 \%$.

Among the six tested fungi, Ph. piricola and S. sclerotiorum were completely inhibited by half of the tested compounds, with an inhibition rate higher than $90 \%$. It was obvious that these derivatives are particularly efficient against the mycelia growth of $P h$. piricola and S. sclerotiorum in vitro. Subsequently, the $\mathrm{EC}_{50} \mathrm{~S}$ of compounds against $\mathrm{Ph}$. piricola and S. sclerotiorum were further evaluated, using cuminaldehyde, thiabendazole, and azoxystrobin as positive controls (Table 1). Cuminaldehyde and thiabendazole showed outstanding antifungal activities against Ph. piricola (25.50 and $21.84 \mu \mathrm{g} \cdot \mathrm{mL}^{-1}$, respectively) and S. sclero-

Table 1. Antifungal activities of titled compounds at $200 \mu \mathrm{g} \cdot \mathrm{mL}^{-1}$ in vitro

\begin{tabular}{|c|c|c|c|c|c|c|}
\hline \multirow{2}{*}{ Compounds } & \multicolumn{6}{|c|}{ Inhibitory (\%) } \\
\hline & B. cinerea & C. lagenarium & Py. oryzae & Ph. piricola & R.solani & S.sclerotiorum \\
\hline $2 a$ & 66.14 & 44.67 & 70.19 & 68.82 & 78.6 & 44.13 \\
\hline $2 b$ & 74.17 & 57.50 & 76.81 & 90.25 & 81.58 & 44.65 \\
\hline $2 c$ & 86.65 & 64.36 & 76.80 & 100 & 93.56 & 37.33 \\
\hline $2 d$ & 100 & 88.85 & 100 & 100 & 100 & 94.42 \\
\hline $2 e$ & 65.72 & 58.83 & 49.74 & 92.01 & 51.14 & 90.18 \\
\hline $2 f$ & 79.49 & 50.91 & 84.74 & 100 & 86.84 & 39.18 \\
\hline $2 \mathrm{~g}$ & 92.84 & 66.20 & 81.20 & 100 & 98.51 & 62.35 \\
\hline $2 \mathrm{~h}$ & 93.68 & 56.00 & 88.26 & 100 & 51.83 & 100 \\
\hline $2 \mathbf{i}$ & 79.07 & 50.91 & 58.77 & 90.00 & 66.59 & 100 \\
\hline $2 \mathbf{j}$ & 66.98 & 48.65 & 47.76 & 79.41 & 73.80 & 55.26 \\
\hline $2 \mathrm{k}$ & 90.33 & 52.04 & 47.37 & 92.52 & 71.73 & 45.83 \\
\hline 21 & 80.33 & 67.89 & 67.43 & 100 & 58.70 & 100 \\
\hline $2-\mathbf{U}^{a)}$ & 64.83 & 45.16 & 53.46 & 73.15 & 68.67 & 48.62 \\
\hline $3 a$ & 51.12 & 54.87 & 50.52 & 70.83 & 60.41 & 100 \\
\hline $3 b$ & 41.94 & 42.98 & 49.34 & 71.34 & 53.21 & 32.43 \\
\hline $3 c$ & 76.98 & 48.65 & 54.45 & 76.89 & 88.22 & 75.05 \\
\hline $3 d$ & 100 & 71.30 & 100 & 100 & 73.80 & 100 \\
\hline $3 e$ & 45.22 & 51.66 & 48.79 & 67.83 & 57.32 & 72.77 \\
\hline $3 f$ & 29.84 & 48.65 & 30.47 & 41.60 & 63.15 & 68.14 \\
\hline $3 g$ & 27.75 & 50.34 & 23.78 & 55.71 & 65.55 & 91.66 \\
\hline $3 \mathrm{~h}$ & 84.35 & 60.43 & 76.53 & 86.36 & 49.70 & 100 \\
\hline $3 \mathbf{i}$ & 55.29 & 48.08 & 48.95 & 80.93 & 31.71 & 86.91 \\
\hline $3 \mathbf{j}$ & 64.47 & 58.26 & 48.95 & 77.90 & 68.31 & 67.18 \\
\hline $3 k$ & 43.61 & 61.67 & 52.10 & 73.35 & 40.50 & 92.86 \\
\hline 31 & 74.64 & 66.82 & 60.28 & 90.07 & 54.45 & 100 \\
\hline $3-\mathbf{U}^{b)}$ & 52.50 & 47.16 & 44.67 & 61.64 & 48.70 & 72.75 \\
\hline Cuminaldehyde & 83.66 & 42.41 & 65.46 & 100 & 70.02 & 68.87 \\
\hline Thiabendazole & 100 & 90.54 & 81.98 & 100 & 100 & 73.95 \\
\hline Azoxystrobin & 88.63 & 70.16 & 83.16 & 75.87 & 72.42 & 95.60 \\
\hline
\end{tabular}

a) Unsubstituted isoxazoline derivatives. ${ }^{b)}$ Unsubstituted pyrazoline drivatives. 
tiorum (63.62 and $44.57 \mu \mathrm{g} \cdot \mathrm{mL}^{-1}$, respectively). Among 24 novel compounds, $\mathbf{2 b}-\mathbf{2 d}, \mathbf{2} \mathbf{f}-\mathbf{2 h}, \mathbf{2}$, and $\mathbf{3 d}$ showed more potential bioactivities against $\mathrm{Ph}$. piricola than those of cuminaldehyde, thiabendazole and azoxystrobin, with the most promising compound $2 \mathrm{~d}$ was 3 -fold more potential than thiabendazole (7.25 vs. $\left.21.84 \mu \mathrm{g} \cdot \mathrm{mL}^{-1}\right)$. Meanwhile, 2d, 2h, 2i, 3d, 3h, 3k, and $3 \mathbf{l}$ showed higher fungicidal activities against $S$. sclerotiorum, and 3d was the most fungicidal compound $\left(12.75 \mu \mathrm{g} \cdot \mathrm{mL}^{-1}\right)$. Among the tested fungi, Ph. piricola is a serious pathogenic fungus on pear and other fruit trees, and S. sclerotiorum is a harmful fungus on various vegetables and crops wordwide. The title compounds showed significantly antifungal activities against these notorious fungi, suggesting that novel compounds have potential and should undergo further evaluation in vivo or in the field.

Against a majority of tested fungi, analysis of the structure and activity relationship (SAR) indicated three general rules: (1) Isoxazolines (2a-2l) were more favorable than pyrazolines (3a31) (Table 1). For instance, the $\mathrm{EC}_{50}$ values of isoxazolines (2b21) against $P h$. piricola ranged from 7.25 to 78.88 , while those of pyrazolines (3a-3l) were higher (Table 2). However, S. sclerotiorum showed an opposite rule. More pyrazolines (3a, 3d, 3g, 3h, $\mathbf{3 k}$, and 31) displayed higher activities than commercial fungicides (thiabendazole and azoxystrobin) did (Table 2). (2) Halogen atoms, as strong electron-withdrawing groups, enhanced the antifungal activities more significantly than methyl group. Generally, the order of activities was $-\mathrm{F}>-\mathrm{Cl}>-\mathrm{Br}>-\mathrm{Me}$, and most of halogen substituted compounds showed higher fungicidal activities than the unsubstituted compounds (2-U and 3-U) (Tables 2). Surprisingly, the compounds containing a fluorine atom (2d, 2h, 2l, 3d, 3h, and 3l) displayed excellent antifungal activities, even higher than commercial fungicides (thiabendazole and azoxystrobin). (3) The Ortho position was more beneficial to antifungal activities than the meta and para positions. Taking fluorine substituent as an example, it was obvious that $\mathbf{2} \mathbf{d}>\mathbf{2 h}>\mathbf{2 l}$ for isoxazoline compounds and $\mathbf{3} \mathbf{d}>\mathbf{3 h}>\mathbf{3 l}$ for pyrazoline compounds (Supplemental Table S2). The rule was also applicable to compounds with $-\mathrm{Cl},-\mathrm{Br}$ and $-\mathrm{Me}$ groups. Therefore, the fungicidal activities of the title compounds closely depended on the core structure, substituent groups, and substituent positions.

Thiabendazole and azoxystrobin were used as positive controls due to their broad-spectrum fungicidal activities. They were also evaluated to be inhibitory against $B$. cinerea and $S$. sclerotiorum in our previous study. ${ }^{18)}$ The present fungicidal activities against the above two fungi were highly coincident with the previous results. Cuminaldehyde, as a main component of spice essential oils, has been proven to inhibit fungi mycelium. ${ }^{1,4-7)}$ In the present study, the cuminaldehyde derivatives showed significant antifungal activities against the tested fungi, and approximately half of the title compounds exhibited higher inhibitory activities than cuminaldehyde per se (Table 1). Notably, 2d showed 3.5 times more activities than cuminaldehyde against Ph. Piricola (7.25 vs. $\left.25.50 \mu \mathrm{g} \cdot \mathrm{mL}^{-1}\right)$, and $3 \mathrm{~d}$ had about 5 times more potential than cuminaldehyde against $S$. sclerotiorum $\left(12.75 v s .63 .62 \mu \mathrm{g} \cdot \mathrm{mL}^{-1}\right)$.
Table 2. $\mathrm{EC}_{50}$ values of compounds $\mathbf{2 a}-\mathbf{2 l}$ and $\mathbf{3 a}-\mathbf{3 l}$ against $P h$. piricola and S. sclerotiorum

\begin{tabular}{|c|c|c|}
\hline \multirow{2}{*}{ Compounds } & \multicolumn{2}{|c|}{$\mathrm{EC}_{50} \pm$ S.E. $\left(\mu \mathrm{g} \cdot \mathrm{mL}^{-1}\right)^{a)}$} \\
\hline & Ph. piricola & S. sclerotiorum \\
\hline $2 a$ & $116.53 \pm 8.16$ & $>200$ \\
\hline $2 b$ & $28.33 \pm 2.48$ & $>200$ \\
\hline $2 c$ & $21.94 \pm 1.59$ & $>200$ \\
\hline $2 d$ & $7.25 \pm 0.64$ & $34.53 \pm 2.42$ \\
\hline $2 e$ & $37.30 \pm 3.34$ & $46.33 \pm 3.77$ \\
\hline $2 \mathrm{f}$ & $22.58 \pm 2.03$ & $>200$ \\
\hline $2 \mathrm{~g}$ & $15.44 \pm 1.25$ & $137.55 \pm 10.35$ \\
\hline $2 h$ & $10.51 \pm 0.84$ & $22.59 \pm 1.50$ \\
\hline $2 \mathbf{i}$ & $48.61 \pm 4.37$ & $16.29 \pm 1.16$ \\
\hline $2 j$ & $78.88 \pm 7.21$ & $158.02 \pm 11.49$ \\
\hline $2 k$ & $33.53 \pm 2.84$ & $>200$ \\
\hline 21 & $13.68 \pm 0.95$ & $20.80 \pm 1.43$ \\
\hline 2-U $\mathbf{U}^{b)}$ & $92.83 \pm 8.37$ & $>200$ \\
\hline $3 a$ & $100.92 \pm 9.44$ & $22.93 \pm 1.58$ \\
\hline $3 b$ & $96.84 \pm 8.63$ & $>200$ \\
\hline $3 c$ & $81.65 \pm 7.40$ & $77.22 \pm 6.65$ \\
\hline $3 d$ & $15.67 \pm 1.18$ & $12.75 \pm 0.88$ \\
\hline $3 e$ & $125.38 \pm 9.77$ & $77.99 \pm 6.83$ \\
\hline $3 \mathrm{f}$ & $>200$ & $141.21 \pm 10.70$ \\
\hline $3 g$ & $171.20 \pm 14.58$ & $33.62 \pm 2.37$ \\
\hline $3 h$ & $60.08 \pm 5.27$ & $20.76 \pm 1.61$ \\
\hline $3 \mathbf{i}$ & $64.52 \pm 5.31$ & $56.29 \pm 4.71$ \\
\hline $3 \mathbf{j}$ & $82.91 \pm 7.59$ & $120.14 \pm 9.43$ \\
\hline $3 k$ & $93.98 \pm 8.58$ & $33.58 \pm 2.59$ \\
\hline 31 & $47.36 \pm 4.05$ & $22.80 \pm 1.60$ \\
\hline $3-U^{c)}$ & $135.67 \pm 10.12$ & $95.37 \pm 8.85$ \\
\hline Cuminaldehyde & $25.50 \pm 1.83$ & $63.62 \pm 5.45$ \\
\hline Thiabendazole & $21.84 \pm 1.73$ & $44.57 \pm 3.36$ \\
\hline Azoxystrobin & $87.59 \pm 7.80$ & $35.22 \pm 2.77$ \\
\hline
\end{tabular}

a) Values are means \pm S.E. of three replicate. ${ }^{b)}$ Unsubstituted isoxazoline derivatives. ${ }^{c}$ Unsubstituted pyrazoline drivatives.

\section{Conclusions}

Two series of novel isoxazoline and pyrazoline compounds derived from cuminaldehyde were designed and synthesized and were characterized via ${ }^{1} \mathrm{H}-\mathrm{NMR},{ }^{13} \mathrm{C}-\mathrm{NMR}$, and HRMS. Results of the preliminary biological activity assay indicated that most of the title compounds showed potential antifungal activities against S. sclerotiorum, Py. oryzae and C. lagenarium and some of the compounds showed potential activities against $R$. solani and B. cinerea. Compound $\mathbf{2} \mathbf{d}$ and $\mathbf{3 d}$, displaying significant fungicidal activities against $S$. sclerotiorum, $P y$. oryzae and $P h$. piricola, are worth being further evaluated in vivo and in the field. Further optimizations of cuminaldehyde derivatives should be carried out to develop more effective antifungal activities. 


\section{Acknowledgements}

This work was supported by Special Finance of Hebei Province (F17C10007 and F17C10008).

\section{References}

1) N. S. Iacobellis, P. Cantore, F. Lo Capasso and F. Senatore: J. Agric. Food Chem. 53, 57-61 (2005).

2) M. J. Tomy, K. V. Dileep, S. Prasanth, D. S. Preethidan, A. Sabu, C. Sadasivan and M. Haridas: Appl. Biochem. Biotechnol. 174, 388-397 (2014).

3) D. Morshedi, F. Aliakbari, A. Tayaranian-Marvian, A. Fassihi, F Pan-Montojo and H. Pérez-Sánchez: J. Food Sci. 80, H2336-H2345 (2015).

4) A. Kedia, B. Prakash, P. K. Mishra and N. K. Dubey: Int. J. Food Microbiol. 168, 1-7 (2014)

5) T. Allahghadri, I. Rasooli, P. Owlia, M. J. Nadooshan, T. Ghazanfari, M. Taghizadeh and S. D. A. Astaneh: J. Food Sci. 75, H54-H61 (2010).

6) J. Wanner, S. Bail, L. Jirovetz, G. Buchbauer, E. Schmidt, V. Gochev, T. Girova, T. Atanasova and A. Stoyanova: Nat. Prod. Commun. 5 , 1355-1358 (2010)

7) J. Tian, X. Q. Ban, H. Zeng, J. S. He, B. Huang and Y. W. Wang: Int. J. Food Microbiol. 145, 464-470 (2011).

8) I. N. Peixoto, H. D. Souza, B. F. Lira, D. F. Silva, E. O. Lima, J. M Barbosa-Filho and P. F. D. Athayde-Filho: J. Braz. Chem. Soc. 27, 1807-1813 (2016).
9) A. Sid, A. Messai, C. Parlak, N. Kazancı, D. Luneau, G. Keşan, L. Rhyman, I. A. Alswaidan and P. Ramasami: J. Mol. Struct. 121, 46-53 (2016).

10) L. A. Tompson and J. A. Ellman: Chem. Rev. 96, 555-600 (1996).

11) T. D. Svejstrup, W. Zawodny, J. J. Douglas, D. Bidgeli, N. S. Sheikh and D. Leonori: Chem. Commun. (Camb.) 52, 12302-12305 (2016).

12) M. P. Sadashiva, K. Mantelingu, S. N. Swamy and K. S. Rangappa: Bioorg. Med. Chem. 11, 4539-4544 (2003).

13) S. Y. Hassan: Molecules 18, 2683-2711 (2013).

14) A. Sid, K. Lamara, M. Mokhtari, N. Ziani and P. Mosset: Eur. J. Chem. 2, 311-313 (2011).

15) V. N. Pathak, R. Joshi, J. Sharma, N. Gupta and V. M. Rao: Phosphorus Sulfur. 184, 1854-1865 (2009).

16) P. L. Zhao, F. Wang, M. Z. Zhang, Z. M. Liu, W. Huang and G. F. Yang: J. Agric. Food Chem. 56, 10767-10773 (2008).

17) C. M. Bhalgat, P. P. Talekar, C. Puttaraj and B. Ramesh: Asian J. Chem. 9, 153-158 (2016)

18) W. J. Si, T. Zhang, Y. F. Li, D. M. She, W. L. Pan, Z. L. Gao, J. Ning and X. D. Mei: J. Pestic. Sci. 41, 15-19 (2015).

19) S. B. Jadhav, R. A. Shastri, K. V. Gaikwad and S. V. Gaikwad: E-J. Chem. 6, S183-S188 (2009).

20) M. Johnson, B. Younglove, L. Lee, R. LeBlanc, H. Holt Jr., P. Hills, H. Mackay, T. Brown, S. L. Mooberry and M. Lee: Bioorg. Med. Chem. Lett. 17, 5897-5901 (2007).

21) K. X. Zhang, M. Y. Dong, X. D. Mei, Y. F. Li, T. Zhang, D. M. She, W. L. Pan, Z. L. Gao and J. Ning: Z. Krist.-New Cryst. St 232, 211-212 (2017). 